\title{
The spectral radius and the maximum degree of irregular graphs
}

\author{
Sebastian M. Cioabă* \\ Department of Mathematics \\ University of California, San Diego \\ La Jolla, CA 92093-0112 \\ scioaba@math.ucsd.edu
}

Submitted: Jan 20, 2007; Accepted: May 3, 2007; Published: May 23, 2007

MR Subject Classifications: 05C50, 15A18

\begin{abstract}
Let $G$ be an irregular graph on $n$ vertices with maximum degree $\Delta$ and diameter $D$. We show that

$$
\Delta-\lambda_{1}>\frac{1}{n D}
$$

where $\lambda_{1}$ is the largest eigenvalue of the adjacency matrix of $G$. We also study the effect of adding or removing few edges on the spectral radius of a regular graph.
\end{abstract}

\section{Preliminaries}

Our graph notation is standard (see West [22]). For a graph $G$, we denote by $\lambda_{i}(G)$ the $i$-th largest eigenvalue of its adjacency matrix and we call $\lambda_{1}(G)$ the spectral radius of $G$. If $G$ is connected, then the positive eigenvector of norm 1 corresponding to $\lambda_{1}(G)$ is called the principal eigenvector of $G$.

The spectral radius of a connected graph has been well studied. Results in the literature connect it with the chromatic number, the independence number and the clique number of a connected graph $[9,11,12,17,23]$. Recently, it has been shown that the spectral radius also plays an important role in modeling virus propagation in networks $[10,21]$.

In this paper, we are interested in the connection between the spectral radius and the maximum degree $\Delta$ of a connected graph $G$. In particular, we study the spectral radius of graphs obtained from $\Delta$-regular graphs on $n$ vertices by deleting a small number of edges or loops. The Erdös-Rényi graph $E R(q)$ is an example of such a graph, see $[9,15]$ and the references within for more details on its spectral radius and other interesting properties.

${ }^{*}$ Research partially supported by an NSERC postdoctoral fellowship. 
It is a well known fact that $\lambda_{1}(G) \leq \Delta(G)$ with equality if and only if $G$ is regular. It is natural to ask how small $\Delta(G)-\lambda_{1}(G)$ can be when $G$ is irregular.

Cioabă, Gregory and Nikiforov [5] proved that if $G$ is an irregular graph on $n$ vertices, with maximum degree $\Delta$ and diameter $D$, then

$$
\Delta-\lambda_{1}>\frac{1}{n\left(D+\frac{1}{n \Delta-2 m}\right)} \geq \frac{1}{n D+n},
$$

where $m$ is the number of edges of $G$. This result improved previous work of Stevanović [20], Zhang [24] and Alon and Sudakov [1].

In [5], the authors conjecture that

$$
\Delta-\lambda_{1}>\frac{1}{n D}
$$

In this paper, we prove this conjecture. Using inequality (1), we improve some recent results of Nikiforov [16] regarding the spectral radius of a subgraph of a regular graph. We also investigate the spectral radius of a graph obtained from a regular graph by adding an edge.

For recent results connecting the spectral radius of a general (not necessarily regular) graph $G$ and that of a subgraph of $G$, see [16].

\section{The spectral radius and the maximum degree}

The following theorem is the main result of this section.

Theorem 2.1. Let $G$ be a connected irregular graph with $n$ vertices, maximum degree $\Delta$ and diameter $D$. Then

$$
\Delta-\lambda_{1}(G)>\frac{1}{n D}
$$

Proof. Let $x$ be the principal eigenvector of $G$. Let $s$ be a vertex of $G$ such that $x_{s}=$ $\max _{i \in[n]} x_{i}$. Since $G$ is not regular, it follows that $x_{s}>\frac{1}{\sqrt{n}}$.

If the degree of $s$ is not $\Delta$, then

$$
\lambda_{1} x_{s}=\sum_{j \sim s} x_{j} \leq(\Delta-1) x_{s}
$$

which implies $\Delta-\lambda_{1} \geq 1>\frac{1}{n D}$ and proves the theorem.

From now on, we will assume that the degree of $s$ is $\Delta$. Our proof is now split in two cases depending on the number of vertices of $G$ whose degree is not $\Delta$ :

Case 1) $G$ contains at least two vertices whose degree is not $\Delta$.

Let $u$ and $v$ be two vertices of $G$ whose degree is not $\Delta$. Let $P: u=i_{0}, i_{1}, \ldots, i_{r}=s$ be a shortest path from $u$ to $s$ in $G$. Obviously, $r \leq D$. Let $Q$ be a shortest path from $v$ to $s$ in $G$. Let $t$ be the smallest index $j$ such that $i_{j}$ is on $Q$. Obviously, $t \in\{0, \ldots, r\}$. 
If $t=0$, then the distance from $u$ to $s$ is at most $D-1$ (this means $r \leq D-1$ ), and applying a similar argument to the one in [5], we obtain that

$$
\begin{aligned}
\Delta-\lambda_{1}(G) & =\Delta \sum_{j=1}^{n} x_{j}^{2}-\sum_{k l \in E(G)} 2 x_{k} x_{l} \\
& =\sum_{i=1}^{n}\left(\Delta-d_{i}\right) x_{i}^{2}+\sum_{k l \in E(G)}\left(x_{k}-x_{l}\right)^{2} \\
& \geq x_{u}^{2}+\sum_{j=0}^{r-1}\left(x_{i_{j+1}}-x_{i_{j}}\right)^{2} \\
& \geq \frac{\left(x_{u}+\sum_{j=0}^{r-1}\left(x_{i_{j+1}}-x_{i_{j}}\right)\right)^{2}}{r+1}=\frac{x_{i_{r}}^{2}}{r+1}>\frac{1}{n D} .
\end{aligned}
$$

If $t \geq 1$, we may assume without any loss of generality that $t=d\left(u, i_{t}\right) \geq d\left(v, i_{t}\right)$. Let $Q_{v, i_{t}}$ denote the sub-path of $Q$ which connects $v$ to $i_{t}$. Using the Cauchy-Schwarz inequality, it follows that

$$
\begin{aligned}
\Delta-\lambda_{1}(G) & =\sum_{i=1}^{n}\left(\Delta-d_{i}\right) x_{i}^{2}+\sum_{k l \in E(G)}\left(x_{k}-x_{l}\right)^{2} \geq x_{u}^{2}+x_{v}^{2}+\sum_{k l \in E(G)}\left(x_{k}-x_{l}\right)^{2} \\
& \geq\left(x_{u}^{2}+\sum_{j=0}^{t-1}\left(x_{i_{j}}-x_{i_{j+1}}\right)^{2}\right)+\left(x_{v}^{2}+\sum_{k l \in E\left(Q_{v, i_{t}}\right)}\left(x_{k}-x_{l}\right)^{2}\right)+\sum_{j=t}^{r}\left(x_{i_{j}}-x_{i_{j+1}}\right)^{2} \\
& \geq \frac{x_{i_{t}}^{2}}{t+1}+\frac{x_{i_{t}}^{2}}{d\left(v, i_{t}\right)+1}+\frac{\left(x_{i_{t}}-x_{s}\right)^{2}}{r-t} \\
& \geq \frac{2 x_{i_{t}}^{2}}{t+1}+\frac{\left(x_{s}-x_{i_{t}}\right)^{2}}{r-t} .
\end{aligned}
$$

The right hand-side is a quadratic function in $x_{i_{t}}$ which attains its minimum when $x_{i_{t}}=$ $\frac{(t+1) x_{s}}{2 r-t+1}$. This implies that

$$
\Delta-\lambda_{1}(G)>\frac{2 x_{s}^{2}}{2 r-t+1} \geq \frac{x_{s}^{2}}{r}
$$

since $t \geq 1$. Because $x_{s}>\frac{1}{\sqrt{n}}$ and $r \leq D$, we obtain

$$
\Delta-\lambda_{1}(G)>\frac{1}{n D} .
$$

This finishes the proof in the case that $G$ has at least two vertices whose degree is not $\Delta$.

Case 2) $G$ contains exactly one vertex whose degree is not $\Delta$.

Let $w$ be a vertex whose principal eigenvector entry is minimum. Then $d_{w}<\Delta$ because

$$
\Delta x_{w}>\lambda_{1} x_{w}=\sum_{j \sim w} x_{j} \geq d_{w} x_{w}
$$


Recall that $x_{s}=\max _{i \in[n]} x_{i}$. Let $\gamma=\frac{x_{s}}{x_{w}}$. We may assume that $\gamma>D$. Otherwise, by summing the equalities $\lambda_{1} x_{i}=\sum_{j \sim i} x_{j}$ over all $i \in[n]$ we have

$$
\Delta-\lambda_{1}=\frac{\left(\Delta-d_{w}\right) x_{w}}{\sum_{i=1}^{n} x_{i}}>\frac{x_{w}}{n x_{s}}=\frac{1}{n \gamma} \geq \frac{1}{n D}
$$

which proves the theorem.

We may also assume that $d(w, s)=D$ because otherwise by applying an argument similar to the one of the previous case, we can easily finish the proof of the theorem.

We claim there exists $j \sim s$ such that $x_{j}<\frac{1}{\sqrt{n}}$. Otherwise, let $j \sim s$ such that $d(j, w)=D-1$. Then applying the argument from the previous case gives

$$
\Delta-\lambda_{1}>\frac{x_{j}^{2}}{D}>\frac{1}{n D}
$$

which finishes the proof of the theorem.

Since $x_{j}<\frac{1}{\sqrt{n}}$ and $j \sim s$, we have

$$
\lambda_{1} x_{s}=\sum_{l \sim s} x_{l}<(\Delta-1) x_{s}+\frac{1}{\sqrt{n}}
$$

which implies

$$
\Delta-\lambda_{1}>1-\frac{1}{x_{s} \sqrt{n}}
$$

If the right-hand side is at least $\frac{1}{n D}$, then we are done. Otherwise, $1-\frac{1}{x_{s} \sqrt{n}}<\frac{1}{n D}$ implies

$$
x_{s}<\frac{D \sqrt{n}}{n D-1}
$$

Since $x_{s}=\max _{i \in[n]} x_{i}, x_{w}=\min _{i \in[n]} x_{i}$, we have that

$$
(n-1) x_{s}^{2}+x_{w}^{2} \geq \sum_{l=1}^{n} x_{l}^{2}=1
$$

which implies

$$
x_{w}^{2} \geq 1-\frac{(n-1) n D^{2}}{(n D-1)^{2}}=\frac{(n D-1)^{2}-(n-1) n D^{2}}{(n D-1)^{2}}=\frac{\left(D^{2}-2 D\right) n+1}{(n D-1)^{2}} .
$$

Assume $D \geq 3$. From (2) and (3), we get that

$$
\gamma^{2}=\frac{x_{s}^{2}}{x_{w}^{2}}<\frac{D^{2} n}{\left(D^{2}-2 D\right) n+1}<D^{2} .
$$

Thus, $\gamma<D$ which is a contradiction with the earlier assumption that $\gamma>D$. This proves the theorem for $D \geq 3$. 
For $D=2$, looking at the square of the adjacency matrix of $G$ we get

$$
\lambda_{1}^{2} x_{s} \leq\left(\Delta^{2}-1\right) x_{s}+x_{w}
$$

which implies

$$
\lambda_{1}^{2} \leq \Delta^{2}-1+\frac{1}{\gamma}<\Delta^{2}-\frac{1}{2}
$$

since $\gamma \geq 2$. Note that inequality (4) holds because there is at least one path of length 2 from $s$ to $w$.

Thus, $\lambda_{1} \leq \sqrt{\Delta^{2}-\frac{1}{2}}<\Delta-\frac{1}{4 \Delta}$.

If $n \geq 2 \Delta$, then we are done. Suppose then that $n<2 \Delta$. Then the vertex $s$ has at least two neighbours at distance 1 from the vertex $w$. We deduce that

$$
\lambda_{1}^{2} x_{s} \leq\left(\Delta^{2}-2\right) x_{s}+2 x_{w}
$$

which implies

$$
\lambda_{1}^{2} \leq \Delta^{2}-2+\frac{2}{\gamma} \leq \Delta^{2}-1 .
$$

Thus,

$$
\lambda_{1} \leq \sqrt{\Delta^{2}-1}<\Delta-\frac{1}{2 \Delta}<\Delta-\frac{1}{n D}
$$

which completes the proof of the theorem.

Because $\lambda_{1}(G)=\Delta(G)$ when $G$ is regular, the following result is an immediate consequence of Theorem 2.1.

Corollary 2.2. Let $G$ be a $\Delta$-regular graph and e be an edge of $G$ such that $G \backslash e$ is connected. Then

$$
\frac{2}{n}>\Delta-\lambda_{1}(G \backslash e)>\frac{1}{n D},
$$

where $D$ is the diameter of $G \backslash e$.

The previous results improve Theorems $4-6$ obtained by Nikiforov in a recent paper [16].

If $f, g: \mathbb{N} \rightarrow[0,+\infty)$ we write $f(n)=O(g(n))$ if there is $c>0$ and $n_{0}>0$ such that $f(n) \leq c g(n)$ for $n \geq n_{0}$ and we write $f(n)=\Theta(g(n))$ if $f(n)=O(g(n))$ and $g(n)=O(f(n))$.

Under the same hypothesis as the previous corollary, if $\Delta \geq 3$ is fixed and $G \backslash e$ is connected, then the diameter $D$ of $G \backslash e$ is at least $\log _{\Delta-1} n+O(1)$. In this case, we obtain the following estimates

$$
\frac{2}{n}>\Delta-\lambda_{1}(G \backslash e)>O\left(\frac{1}{n \log _{\Delta-1} n}\right) .
$$

It seems likely that the upper bound gives the right order of magnitude for $\Delta-\lambda_{1}(G \backslash e)$, but proving this fact is an open problem. 
If $\Delta=\Theta(n)$ and $G \backslash e$ is connected, then the diameter $D$ of $G \backslash e$ is $O(1)$. To see this consider a path $i_{0}, \ldots, i_{D}$ of length $D$ in $G \backslash e$. For $0 \leq j \leq D$, let $N_{j}$ denote the neighborhood of vertex $j$ in $G \backslash e$. It follows that for $j \equiv 0(\bmod 3), 0 \leq j \leq D$, the sets $N_{j}$ are pairwise disjoint. Also, for all but at most two $j$ 's, we have $\left|N_{j}\right|=\Delta$. These facts imply that

$$
n \geq \sum_{j \equiv 0}\left|N_{j}\right|>\left(\frac{D+1}{3}-2\right) \Delta+2(\Delta-1)=\frac{\Delta(D+1)}{3}-2 .
$$

Thus,

$$
D<\frac{3(n+2)}{\Delta}=O(1)
$$

Hence, in this case, our estimates imply that

$$
\Delta-\lambda_{1}(G \backslash e)=\Theta\left(\frac{1}{n}\right)
$$

Note that the previous argument can be also used to show that if $\Delta=\Theta(n)$ and $H$ is a connected graph obtained from a $\Delta$-regular graph on $n$ vertices by deleting a constant number of edges, then

$$
\Delta-\lambda_{1}(H)=\Theta\left(\frac{1}{n}\right)
$$

\section{Adding an edge to a regular graph}

In this section, we analyze the effect of adding an edge on the spectral radius of a regular graph. We need different techniques in this case because the spectral radius will be closer to the minimum degree than to the maximum degree of the graph.

Theorem 3.1. Let $H$ be a connected, k-regular graph and $e \notin E(H)$. If $G$ is the graph obtained from $H$ by adding the edge $e$ and $k-\lambda_{2}(H)>1$, then

$$
\frac{2}{n} \cdot\left(1+\frac{1}{k-\lambda_{2}(H)-1}\right)>\lambda_{1}(G)-\lambda_{1}(H)>\frac{2}{n} \cdot\left(1+\frac{1}{2(k+2)}\right) .
$$

Proof. The lower bound follows by applying the following result obtained by Nikiforov [18]. See also [4] for related results.

Theorem 3.2. Let $G$ be an irregular graph with $n$ vertices and $m$ edges having maximum degree $\Delta$. If $G$ has at least two vertices of degree $\Delta$ and at least two vertices of degree less than $\Delta$, then

$$
\lambda_{1}(G)>\frac{2 m}{n}+\frac{1}{2 m+n}
$$

For the upper bound, we use the following result of Maas [14] (see also Theorem 6.4.1 in [6] and [3]). 
Theorem 3.3 (Maas [14]). Let $x$ be the principal eigenvector of a graph $H$ and let $i$ and $j$ be two non-adjacent vertices in $H$. Then

$$
\lambda_{1}(H+i j)-\lambda_{1}(H)<1+\delta-\beta,
$$

where

$$
\beta=\lambda_{1}(H)-\lambda_{2}(H)
$$

and $\delta$ satisfies the equation

$$
\frac{\delta(1+\delta)(2+\delta)}{\left(x_{i}+x_{j}\right)^{2}+\delta\left(2+\delta+2 x_{i} x_{j}\right)}=\beta .
$$

Applying the previous theorem and using the fact that the principal eigenvector of $H=G \backslash e$ has all entries equal to $\frac{1}{\sqrt{n}}$, we obtain that

$$
\left.\lambda_{1}(G)-\lambda_{1}(H)\right)<1+\delta-\beta=\frac{2 \beta}{\delta n} .
$$

If $k-\lambda_{2}(H)=\beta>1$, we obtain that

$$
\beta=\frac{\delta(1+\delta)(2+\delta)}{(\delta+2)\left(\delta+\frac{2}{n}\right)}=\frac{\delta(1+\delta)}{\delta+\frac{2}{n}}<1+\delta .
$$

Thus, $\frac{2 \beta}{\delta}<\frac{2 \beta}{\beta-1}=\frac{2\left(k-\lambda_{2}(H)\right)}{k-\lambda_{2}(H)-1}$. Hence, we deduce that

$$
\lambda_{1}(G)-\lambda_{1}(G \backslash e)<\frac{2\left(k-\lambda_{2}(H)\right)}{\left(k-\lambda_{2}(H)-1\right) n}
$$

which proves the theorem.

For $k \geq 3$ fixed and $\epsilon>0$, Friedman [8] proved that most $k$-regular graphs $H$ have $\lambda_{2}(H) \leq 2 \sqrt{k-1}+\epsilon$. This implies that for most $k$-regular graphs $H$ and for each $e \notin E(H)$, we have

$$
\lambda_{1}(H+e)-\lambda_{1}(H)=\Theta\left(\frac{1}{n}\right) .
$$

If $H$ is a $k$-regular graph with $k-\lambda_{2}(H) \leq 1$, then (7) might not hold. This is true at least for $k=2$ as seen by the graph $G_{n}$ on $n$ vertices which is obtained from a cycle on $n$ vertices by adding an edge between two vertices at distance 2 . It follows from the work of Simić and Kocić [19] (see also [6] equation (3.4.5) on page 63) that

$$
\lim _{n \rightarrow \infty} \lambda_{1}\left(G_{n}\right)=2.3829
$$

while $\lambda_{1}\left(C_{n}\right)=2$, so clearly $\lambda_{1}\left(G_{n}\right)-\lambda_{1}\left(C_{n}\right) \neq \Theta\left(\frac{1}{n}\right)$. 


\section{Final Remarks}

It is worth mentioning that there are infinite families of irregular graphs with maximum degree $\Delta$ such that $\Delta-\lambda_{1} \leq \frac{c}{n D}$, where $c$ is an absolute constant. Cioabă, Gregory and Nikiforov [5] describe such a family with $c=4 \pi^{2}$ while Liu, Shen and Wang [13] found an infinite family with $c=3 \pi^{2}$. It has yet to be determined what the best such constant $c$ can be for all $n$ and $D$.

Note that the argument of Theorem 2.1 can be extended easily to multigraphs. From the proof of Theorem 2.1, it is easy to see that if one can find better upper bounds for the distance between vertices corresponding to the extreme entries of the principal eigenvector of an irregular graph $G$, then one can improve the result of Theorem 2.1. However, there are infinite families of irregular graphs for which the distance between such vertices equals the diameter. We describe one such family in the next paragraph.

For $k \geq 2$, consider the cycle on $n=2 k+1$ vertices with vertex set $\{1, \ldots, 2 k+1\}$ and edges $\{i, i+1\}$ for $1 \leq i \leq 2 k$ and $\{2 k+1,1\}$. Add the edges $\{k, k+2\},\{k+1, k+3\}$ and for $k \geq 3$, the edges $\{i, 2 k+3-i\}$ for $2 \leq i \leq k-1$. The resulting graph $G$ has maximum degree 3 and 1 is the only vertex whose degree is 2 . It can be shown easily by induction that if $x$ is the principal eigenvector of $G$, then $x_{1}=\min _{i \in[n]} x_{i}$ and $x_{k+1}=x_{k+2}=\max _{i \in[n]} x_{i}$. Note that $d(1, k+1)=d(1, k+2)=k$ which equals the diameter of $G$.

When $n=7$, the graph obtained by the above procedure is shown in Figure 1 with its vertices labeled by their entries in the principal eigenvector. Note that although the distance between vertices corresponding to extreme entries of the principal eigenvector equals the diameter, there are vertices whose eigenvector entry is at least $\frac{1}{\sqrt{7}}=0.378$ which are at distance less than the diameter from the vertex whose eigenvector entry is minimum. If this fact would be true for any irregular graph, it would imply Theorem 2.1.

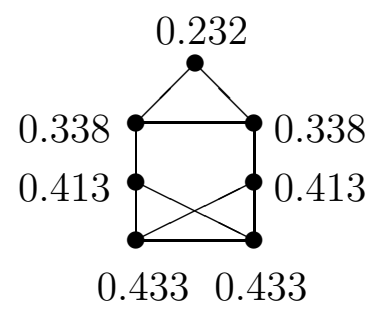

Figure 1: An irregular graph and its principal eigenvector

It would be interesting to determine the precise asymptotic behaviour of the spectral radius of a graph obtained from a $\Delta$-regular graph on $n$ vertices by deleting an edge when $\Delta=o(n)$. Another problem of interest is to find the exact asymptotic behaviour of the spectral radius of a graph obtained from a $k$-regular graph $H$ by adding an edge when $k=\Theta(n)$ or when $k-\lambda_{2}(H) \leq 1$.

A slightly different direction of research was taken by Biyikoglu and Leydold in [2] where they study the graphs which have the maximum spectral radius in the set of all 
connected graphs with given degree sequence. In particular, the authors show that the maximum is increasing with respect to the majorization order. Even for graphs with simple degree sequences, determining the maximum spectral radius seems a nontrivial problem.

\section{Acknowledgments}

I am grateful to Steve Butler, David Gregory and the anonymous referee for their careful reading of the paper and I thank Fan Chung, Orest Bucicovschi and Vlado Nikiforov for helpful discussions.

\section{References}

[1] N. Alon and B. Sudakov, Bipartite subgraphs and the smallest eigenvalue, Combin. Probab. Comput., 9 (2000), no. 1, 1-12.

[2] T. Biyikoglu and J. Leydold, Largest eigenvalue of degree sequences, available at http: //arxiv.org/abs/math.CO/0605294

[3] Z. Bo, The changes in indices of modified graphs, Linear Algebra and its Applications, 356 (2002), 95-101.

[4] S. M. Cioabă and D. A. Gregory, Large matchings from eigenvalues, Linear Algebra and its Applications, 422 (2007), 308-317.

[5] S. M. Cioabă, D. A. Gregory and V. Nikiforov, Extreme eigenvalues of nonregular graphs, J. Combin. Theory, Series B, 97 (2007), 483-486.

[6] D. Cvetković, P. Rowlinson and S. Simić, Eigenspaces of graphs, Encyclopedia of Mathematics, Cambridge University Press, 1997.

[7] E. R. van Dam and R. E. Kooij, The minimal spectral radius of graphs with a given diameter, Linear Algebra and its Applications, 423 (2007), 408-419.

[8] J. Friedman, A proof of Alon's second eigenvalue conjecture, Memoirs of the AMS, to appear.

[9] C. Godsil and M. Newman, Eigenvalue bounds for independent sets, J. Combin. Theory, Series B, to appear.

[10] A. Jamakovic, R. E. Kooij, P. Van Mieghem, E. R. van Dam, Robustness of networks against viruses: the role of the spectral radius, Proceedings of the 13th Annual Symposium of the IEEE/CVT Benelux, Liége, Belgium

[11] W. Haemers, Interlacing eigenvalues and graphs, Linear Algebra and its Applications, 226/228 (1995), 593-616.

[12] A. J. Hoffman, On eigenvalues and colorings of graphs. In Graph theory and its Applications (Proc. Advanced Sem., Math. Research Center, Univ. of Wisconsin, Madison, Wisc., 1969), 79-91, Academic Press, 1970. 
[13] B. Liu, J. Shen and X. Wang, On the largest eigenvalue of non-regular graphs, J. Combin. Theory, Series B, to appear.

[14] C. Maas, Perturbation results for the adjacency spectrum of a graph, Z. angew Math. Mech., 67 (1987), 428-430.

[15] M. Newman, Independent Sets and Eigenspaces, Ph.D. Thesis, University of Waterloo (2004).

[16] V. Nikiforov, Revisiting two classical results in graph spectra, The Electronic Journal of Combinatorics, Volume 14 (2007), R14.

[17] V. Nikiforov, Some inequalities for the largest eigenvalue of a graph, Combinatorics, Probability and Computing 11 (2001), 179-189.

[18] V. Nikiforov, Bounds on graph eigenvalues II, available at http: //arxiv.org/abs/math.CO/0612461

[19] S. Simić and V. Kocić, On the largest eigenvalue of some homeomorphic graphs, Publ. Inst. Math. (Beograd), 40 (54) (1986), 3-9.

[20] D. Stevanović, The largest eigenvalue of nonregular graphs, J. Combin. Theory Ser. $B, 91$ (2004), no. 1, 143-146.

[21] Y. Wang, D. Chakrabarti, C. Wang and C. Faloutsos, Epidemic spreading in real networks: An eigenvalue viewpoint, 22nd Symposium in Reliable Distributed Computing, Florence, Italy, Oct. 6-8, 2003.

[22] D. B. West, Introduction to graph theory, Prentice-Hall, New Jersey, 2nd Edition, (2001).

[23] H. Wilf, Spectral bounds for the clique and independence number of graphs, $J$. Combin. Theory, Ser. B 40 (1986), 113-117.

[24] X.-D. Zhang, Eigenvectors and eigenvalues of non-regular graphs, Linear Algebra and its Applications, 409 (2005), 79-86. 